Please send trade news information and illustrations to Terry Mordecai at the BDJ, 64 Wimpole Street, London W1G 8YS. Trade news is supplied as a service to the reader and does not imply endorsement by the BDJ. Normal and prudent research should be exercised before purchase of use of any product mentioned.

\title{
Faster and whiter
}

Prestige Dental is pleased to announce the launch of the Remecure, which they claim is a fast curing and whitening lamp based on plasma technology. The entire curing takes between three and nine seconds compared with over six minutes using conventional halogen and LED technologies.

Prestige Dental claims that once the patient's teeth have been cleaned and the gel is applied, the lamp is activated and the tip is placed in the front of the patient's mouth. The lamp burns one minute, stops for 30 seconds and continues this process for 10 minutes.

They also claim the comfort for the dentist is increased considerably using this process. For more information phone 0800 $591175 . \quad$ Reader response number 52
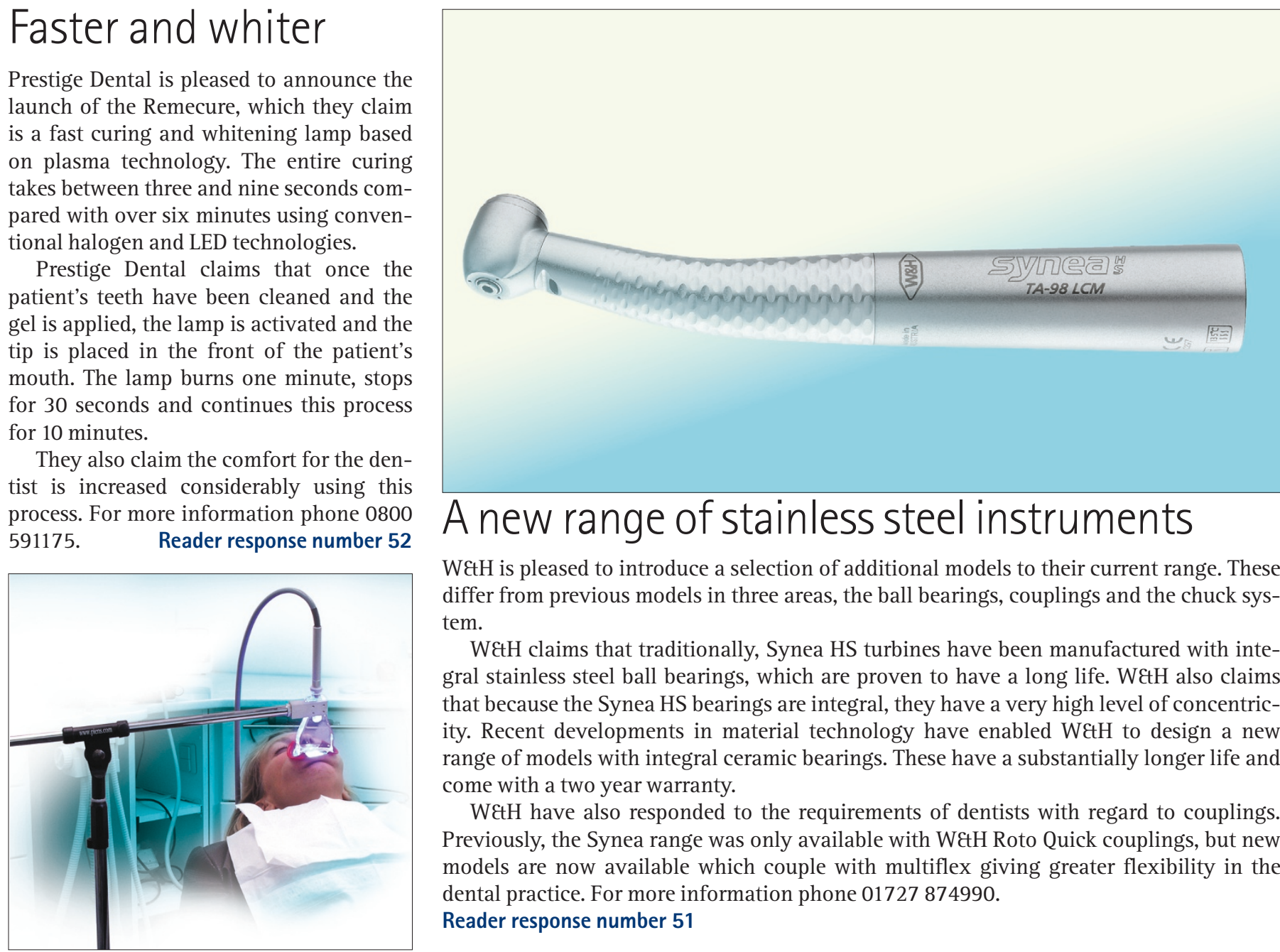

\section{A new range of stainless steel instruments}

WEH is pleased to introduce a selection of additional models to their current range. These differ from previous models in three areas, the ball bearings, couplings and the chuck system.

WEH claims that traditionally, Synea HS turbines have been manufactured with integral stainless steel ball bearings, which are proven to have a long life. W\&H also claims that because the Synea HS bearings are integral, they have a very high level of concentricity. Recent developments in material technology have enabled W\&H to design a new range of models with integral ceramic bearings. These have a substantially longer life and come with a two year warranty.

WEH have also responded to the requirements of dentists with regard to couplings. Previously, the Synea range was only available with WEH Roto Quick couplings, but new models are now available which couple with multiflex giving greater flexibility in the dental practice. For more information phone 01727874990.

Reader response number 51

\section{Two new updated toothbrushes from Oral-B}

Oral-B claims dental professionals estimate that up to 70\% of people do not brush their teeth adequately and that many people find it difficult to apply a proper brushing technique and most do not brush long enough to thoroughly remove plaque.

The latest innovation from Oral-B tackles both these issues. Professional Care 7000 is an upgrade on the 3D Excel power toothbrush. The new brush has fifteen percent more oscillations per minute than its predecessor, which Oral-B claims leaves teeth feeling exceptionally clean.

The Professional Care 7000 also signals (both auditory and sensory) after thirty seconds to encourage patients to clean quadrant-by-quadrant. Oral-B also claims the aesthetics have also been enhanced, and the brush is now available in a blue translucent colour with a contrasting lighter blue rubber thumb grip.

There are two models in the Professional 7000 series, the 7500DLX and the 7000. Oral-B also claims both offer the same outstanding performance, however, the former has an additional replacement head as well as two Interspace brush heads for interdental cleaning. For more information phone 02088477800.

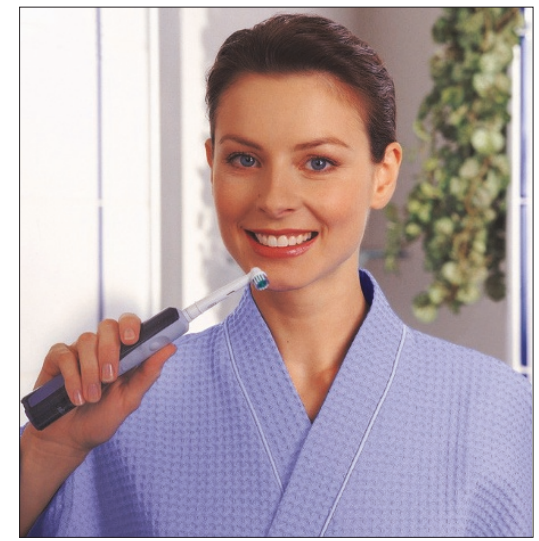

